\title{
A subset of circulating microRNAs is expressed differently in patients with myocardial infarction
}

\author{
JIANQIU LIANG ${ }^{1,2}$, SHUCHANG BAI ${ }^{2}$, LIANG SU ${ }^{1}$, CHEN LI $^{2}$, JIANDI WU ${ }^{2}$, \\ ZHANGQING XIA ${ }^{2}$ and DINGLI XU ${ }^{1}$ \\ ${ }^{1}$ Department of Cardiology, Nanfang Hospital, Southern Medical University, Guangzhou, Guangdong 510515; \\ ${ }^{2}$ Department of Cardiology, Foshan Second People's Hospital, Foshan, Guangdong 528000, P.R. China
}

Received June 21, 2013; Accepted March 11, 2014

DOI: $10.3892 / \mathrm{mmr} .2015 .3422$

\begin{abstract}
The objective of the present study was to analyze the differences in the plasma microRNA (miRNA) expression profiles between patients with myocardial infarction (MI) (with or without heart failure) and individuals in a normal control group using an miRNA array. Specific miRNAs were selected to explore novel circulating markers for MI and heart failure. A total of 15 patients with heart failure and 10 patients without heart failure following acute MI (AMI) were recruited as the AMI with heart failure (AMHF) and with no heart failure (AMNHF) groups, respectively. In addition, 10 patients with an older ( $\geq 1$ year) MI with heart failure were selected as the old MI and heart failure (OMHF) group. Finally, 10 patients with normal coronary angiograms were recruited as the control (N) group. The plasma of peripheral venous blood was collected for miRNA array detection. In the AMHF group, the expression of 17 miRNAs was upregulated and the expression of 21 miRNAs was downregulated by $>1.5$-fold compared with that in the AMNHF group. Compared with the $\mathrm{N}$ group, the expression of miRNAs in the AMNHF group was upregulated in 38 and downregulated in 48 cases by $>1.5$-fold. Compared with the OMHF group, 13 miRNAs were upregulated and 43 were downregulated by $>1.5$-fold in the AMHF group. Significant differences in the miRNA expression profiles were observed between patients with different stages of heart failure following MI and individuals in the normal control group. These differences were determined using miRNA array analysis methods based on the peripheral blood plasma. Thus, the specific miRNAs identified in this study may be novel circulating markers for MI and heart failure.
\end{abstract}

Correspondence to: Dr Dingli Xu, Department of Cardiology, Nanfang Hospital, Southern Medical University, 1838 North Guangzhou Road, Guangzhou, Guangdong 510515, P.R. China E-mail: xud1589@163.com

Key words: myocardial infarction, heart failure, plasma, miRNA, miRNA array

\section{Introduction}

Cardiovascular disease is one of the major causes of death worldwide. The pathologies of cardiovascular disease are complex, although the most important are inflammation and oxidative stress. Biomarkers of inflammation and oxidative stress may serve to help identify patients at risk for cardiovascular disease, to monitor the efficacy of treatments, and to develop novel pharmacological tools. The use of serological biomarkers may improve clinical decision making and a therapeutic strategy setting. For primary cardiovascular events, markers with strong predictive potential are mainly associated with lipids. For secondary cardiovascular events, markers are associated more with ischemia. However, there are fewer studies on microRNAs (miRNAs), which as cardiovascular biomarkers. miRNAs are small, endogenous, noncoding RNAs that regulate gene expression by targeting the degradation or translational repression of mRNA. Previously, it has been demonstrated that miRNAs circulating in the bloodstream are useful biomarkers for cardiovascular disease (1-3).

Wang et al (4) reported that miR-208a is an excellent diagnostic marker for acute myocardial infarction (AMI), which was demonstrated by its sensitivity in detecting AMI in patients within $4 \mathrm{~h}$ of the onset of symptoms. Kuwabara et al (5) reported that circulating miR-133a serves as a useful marker for cardiomyocyte death and can thus be used for the detection of several cardiovascular diseases, including AMI, unstable angina and takotsubo cardiomyopathy.

Few reports, however, have analyzed the differences in miRNA expression profiles between patients with MI (with or without heart failure) and individuals in a normal control group using an miRNA array. Therefore, the aim of the present study was to investigate specific miRNAs that were differentially expressed in patients with MI (with or without heart failure) and individuals in a normal control group. The results were used as a basis for exploring novel circulating markers for MI and heart failure.

\section{Materials and methods}

Patients. A total of 25 consecutive patients, whose symptoms were consistent with the established diagnostic criteria for AMI, were selected and divided into two groups: AMI with 
Table I. Baseline characteristics of each group.

\begin{tabular}{|c|c|c|c|c|c|}
\hline Variable & AMNHF & AMHF & OMHF & Control & P-value \\
\hline Age (years) & $59.2 \pm 6.0$ & $60.5 \pm 6.6$ & $58.7 \pm 7.3$ & $58.4 \pm 6.5$ & 0.852 \\
\hline Gender, M/F (n/n) & $6 / 4$ & $8 / 7$ & $6 / 6$ & $5 / 5$ & - \\
\hline $\operatorname{BMI}\left(\mathrm{kg} / \mathrm{m}^{2}\right)$ & $24.00 \pm 2.38$ & $24.58 \pm 2.55$ & $24.10 \pm 3.43$ & $23.86 \pm 1.64$ & 0.904 \\
\hline $\mathrm{SBP}(\mathrm{mmHg})$ & $125.8 \pm 5.1$ & $120.8 \pm 5.6$ & $122.5 \pm 4.2$ & $121.9 \pm 4.9$ & 0.123 \\
\hline$\beta$-blocker, Y/N (n/n) & $10 / 0$ & $4 / 15$ & $10 / 0$ & $0 / 10$ & - \\
\hline ACEI, Y/N (n/n) & $9 / 10$ & $15 / 15$ & $8 / 2$ & $0 / 10$ & - \\
\hline $\mathrm{ARB}, \mathrm{Y} / \mathrm{N}(\mathrm{n} / \mathrm{n})$ & $1 / 10$ & $0 / 15$ & $2 / 8$ & $0 / 10$ & - \\
\hline Statins, Y/N (n/n) & $10 / 10$ & $10 / 0$ & $10 / 0$ & $1 / 9$ & - \\
\hline
\end{tabular}

AMNHF, acute myocardial infarction with no heart failure; AMHF, acute myocardial infarction with heart failure; OMHF, old myocardial infarction with heart failure; BMI, body mass index; SBP, systolic blood pressure; ACEI, angiotensin-converting enzyme inhibitor; ARB, angiotensin receptor blocker; $\mathrm{M}$, male; F, female; Y, yes; N, no.

no heart failure [AMNHF; Killip class I and left ventricular ejection fraction (LVEF) $>50 \%$ ] and the AMI with heart failure (AMHF; Killip class $\geq$ II and LVEF $\leq 40 \%$ ) groups. A total of 10 patients who had presented with AMI one year before the study was conducted were recruited as the old MI with heart failure (OMHF) group ( $\mathrm{OVEF}>50 \%)$. For the control (N) group, 10 patients with normal coronary angiography results and an LVEF $>50 \%$ were selected. The present study was approved by the ethics committee of Foshan Second People's Hospital (Foshan, China). Written informed consent was obtained from the patients.

Plasma collection. Fasting blood samples were collected into EDTA anticoagulant separator tubes, which were subsequently centrifuged at $1,000 \mathrm{x} \mathrm{g}$ for $10 \mathrm{~min}$ at $4^{\circ} \mathrm{C}$ to separate the clots. The plasma was then removed from the tubes and stored at $-80^{\circ} \mathrm{C}$ until the time of the assay.

RNA isolation and miRNA analysis. Total RNA was isolated using TRIzol ${ }^{\circledR}$ reagent (Invitrogen Life Technologies, Carlsbad, CA, USA) and the miRNeasy Mini kit (Qiagen, Hilden, Germany) in accordance with the manufacturer's instructions. All RNA species were efficiently recovered using this method, including miRNA. The quality and quantity of the isolated RNA was evaluated using a nanodrop spectrophotometer (ND-1000; Nanodrop Technologies, Waltham, MA, USA), and gel electrophoresis was utilized to assess the integrity of the RNA.

Following RNA isolation from the samples, miRNA labeling was conducted with the miRCURY ${ }^{\mathrm{TM}} \mathrm{Hy} 3^{\mathrm{TM}} / \mathrm{Hy}^{\mathrm{TM}}$ Power Labeling kit (Exiqon A/S, Vedbaek, Denmark) in accordance with the manufacturer's instructions. The 3'-end of each $1-\mu \mathrm{g}$ sample was subsequently labeled with a Hy3 fluorescent label, using T4 RNA ligase. In brief, RNA in $2.0 \mu$ l water was combined with $1.0 \mu \mathrm{l}$ calf intestinal alkaline phosphatase (CIP) buffer and CIP. This mixture was then incubated for $30 \mathrm{~min}$ at $37^{\circ} \mathrm{C}$, prior to the reaction being terminated by incubation for $5 \mathrm{~min}$ at $95^{\circ} \mathrm{C}$. A total of $3.0 \mu \mathrm{l}$ labeling buffer, $1.5 \mu \mathrm{l}$ fluorescent label (Hy3), $2.0 \mu \mathrm{l}$ dimethylsulfoxide and $2.0 \mu \mathrm{l}$ labeling enzyme were subsequently added to the mixture. The labeling reaction was incubated for $1 \mathrm{~h}$ at $16^{\circ} \mathrm{C}$, and the termination of the reaction was achieved by incubation for $15 \mathrm{~min}$ at $65^{\circ} \mathrm{C}$.
Following the termination of the labeling procedure, the Hy3-labeled samples were hybridized on the miRCURY ${ }^{\mathrm{TM}}$ Locked Nucleic Acid array (v. 16.0; Exiqon A/S) according to the manufacturer's instructions. The $25-\mu 1$ mixture from the Hy3-labeled samples was then combined with $25 \mu$ l hybridization buffer for denaturation for $2 \mathrm{~min}$ at $95^{\circ} \mathrm{C}$. Following incubation on ice for $2 \mathrm{~min}$, the mixture was hybridized to the microarray for $16-20 \mathrm{~h}$ at $56^{\circ} \mathrm{C}$ in a 12 -Bay Hybridization System (Nimblegen Systems, Inc., Madison, WI, USA). This procedure was designed to improve the uniformity of the hybridization and enhance the signal by performing active mixing and maintaining a constant incubation temperature. Once hybridization was complete, the slides were washed multiple times using a wash buffer kit (Exiqon A/S), prior to being dried by centrifugation for $5 \mathrm{~min}$ at $200 \mathrm{x} \mathrm{g}$. The Axon GenePix 4000B microarray scanner (Axon Instruments, Foster City, CA, USA) was subsequently utilized to scan the slides.

Volcano plots. Volcano plots enable the visualization of differential expression between two different groups. Fold-change and $\mathrm{P}$-values are utilized to create the plots, thus facilitating the visualization of the correlation between the fold-change (the magnitude of the change) and the statistical significance (which takes the magnitude of the change and variability into consideration). These plots also allow subsets of genes to be isolated based on different values.

Statistical analysis. The scanned images were imported into GenePix Pro 6.0 software (Axon Instruments) for grid alignment and data extraction. The replicated miRNAs were averaged and miRNAs with intensities of $\geq 50$ in all samples were selected for the calculation of the normalization factor. Expressed data were normalized using median normalization. Following normalization, significant differences in miRNA expression were identified using volcano plot filtering, and hierarchical clustering was performed using MultiExperiment Viewer 4.6 software (The Institute for Genomic Research; http://www.tigr.org/software/tm4/mev.html). P<0.05 was considered to indicate a statistically significant difference between values. 
A

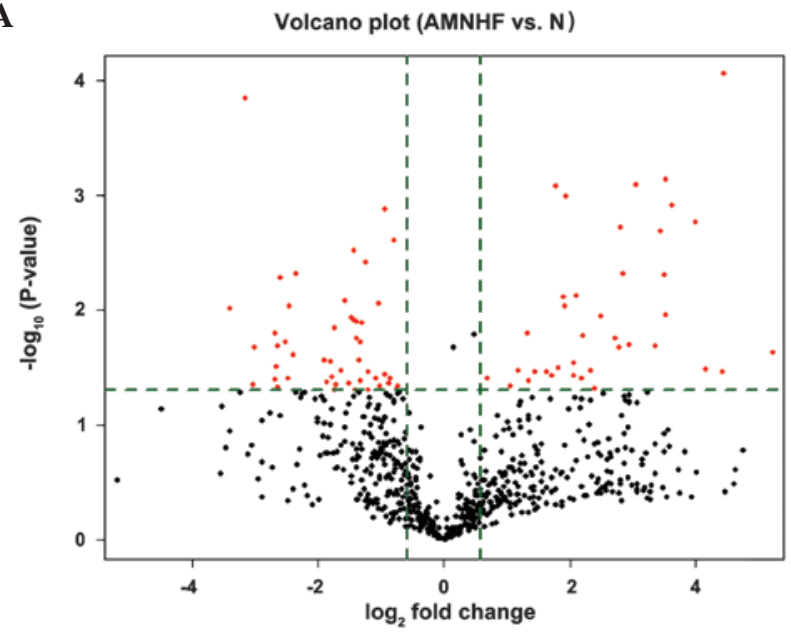

B

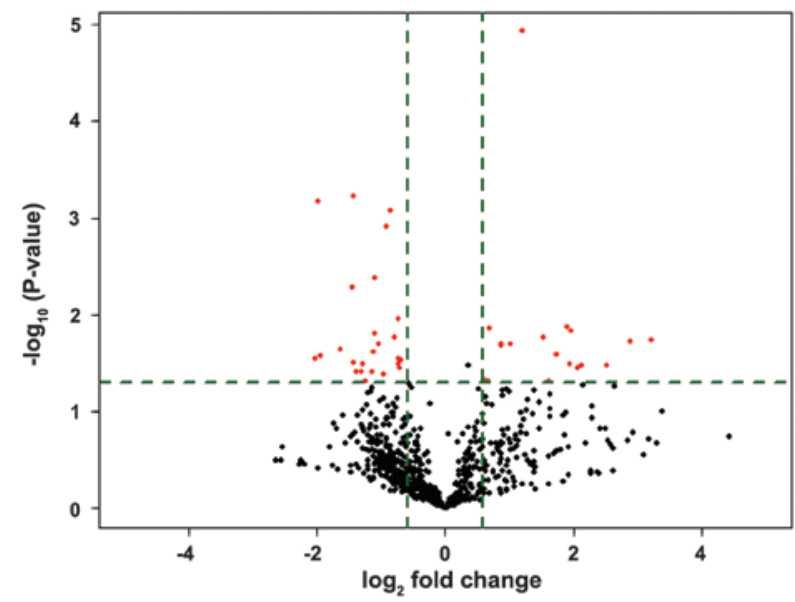

C

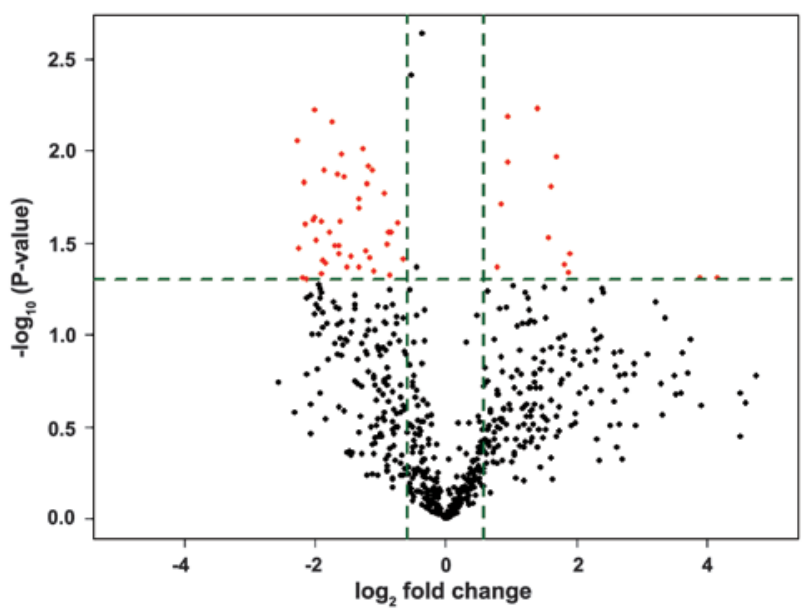

Figure 1. Volcano plots of differentially expressed miRNAs for the (A) AMNHF versus the N group; (B) AMHF versus the AMNHF group; (C) AMHF versus the OMNHF group. Red dots represent the differentially expressed miRNAs with statistical significance. AMNHF, acute myocardial infarction with no heart failure; AMHF, acute myocardial infarction with heart failure; OMNHF, old myocardial infarction with no heart failure; $\mathrm{N}$, control; miRNA, microRNA.

\section{Results}

Baseline characteristics. No significant differences were observed in age, gender, body mass index or systolic blood pressure among the groups $(\mathrm{P}>0.05)$. The majority of the participants in the patient groups were taking angiotensin-converting enzyme inhibitors (ACEIs), angiotensin receptor blockers (ARBs), statins or $\beta$-blockers. Only one participant in the $\mathrm{N}$ group used statins and none of them were taking ACEIs/ARBs or $\beta$-blockers (Table I).

Volcano plots. In Fig. 1, the vertical lines correspond to 1.5 -fold positive and negative differences, respectively, and the horizontal lines represent a P-value of 0.05. Consequently, the red dots in the plot represent the differentially expressed miRNAs with statistical significance.

Heat map and hierarchical clustering. The heat map diagram shows the result of the two-way hierarchical clustering of the miRNAs and samples. Each row represents an miRNA and each column represents a sample. The miRNA clustering tree is shown on the left and the sample clustering tree appears at the top. The color scale shown at the top illustrates the relative expression level of a miRNA in a specific slide: Red represents a high relative expression level and green represents a low relative expression level (Fig. 2).

Differential expression of miRNAs. In the AMHF group, the expression of $17 \mathrm{miRNAs}$ was upregulated and that of 21 miRNAs was downregulated by $>1.5$-fold as compared with the expression in the AMNHF group. Compared with the $\mathrm{N}$ group, the expression of miRNAs in the AMNHF group was upregulated in 38 and downregulated in 48 cases by $>1.5$-fold. Compared with the OMHF group, 13 miRNAs were upregulated and 43 were downregulated by $>1.5$-fold in the AMHF group (Fig. 1).

To confirm the results from the discovery phase, the expression levels of candidate miRNAs were examined by quantitative polymerase chain reaction (qPCR) using a 7900HT Fast Real-Time PCR system (Life Technologies, Grand Island, NY, USA). Compared with expression in the AMNHF group, the expression of hsa-miR-493-5p, hsa-miR-369-3p, hsa-miR-495, hsa-miR-3615 and hsa-miR-433 was upregulated, and that of hsa-miR-877-3p, hsa-miR-1306-3p, hsv1-miR-H2, hsa-miR-3130-5p and hcmv-miR-UL22A was downregulated in the AMHF group $(\mathrm{P}<0.05)$. In the AMNHF group, the expression of hsa-miR-596, hsa-miR-657, hsa-miR-1306-3p, hsv1-miR-H2 and hsa-miR-3130-5p was upregulated, and the expression of hsa-miR-409-5p, hsa-miR-24-2-5p, hsa-miR-335-5p, hsa-miR-19a-3p and hsa-miR-130a-3p was downregulated compared with that in the $\mathrm{N}$ group $(\mathrm{P}<0.05)$. Compared with the expression in the OMHF group, the expression of hsa-miR-338-5p, hsa-miR-124-3p, hsa-miR-214-3p, hsa-miR-433 and hsa-miR-19a-3p was upregulated, and that of hsv2-miR-H24, hsa-miR-1249, hsa-miR-4258, hsa-miR-1470 and hsa-miR-2110 was downregulated in the AMHF group $(\mathrm{P}<0.05)$.

\section{Discussion}

To the best of our knowledge, this is the first study that analyzes the differences in the miRNA expression profile between patients with MI (with or without heart failure) and a normal control group using a plasma miRNA array. 
A

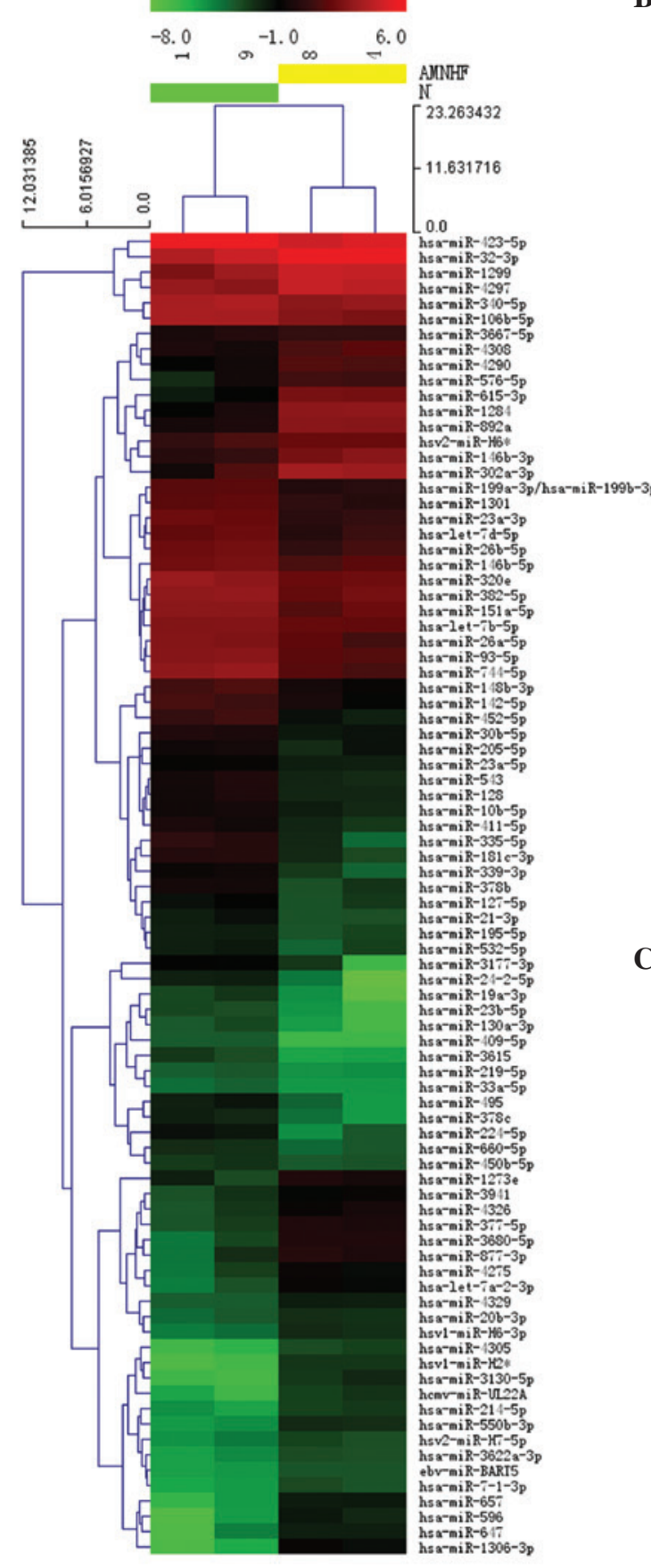

C
B
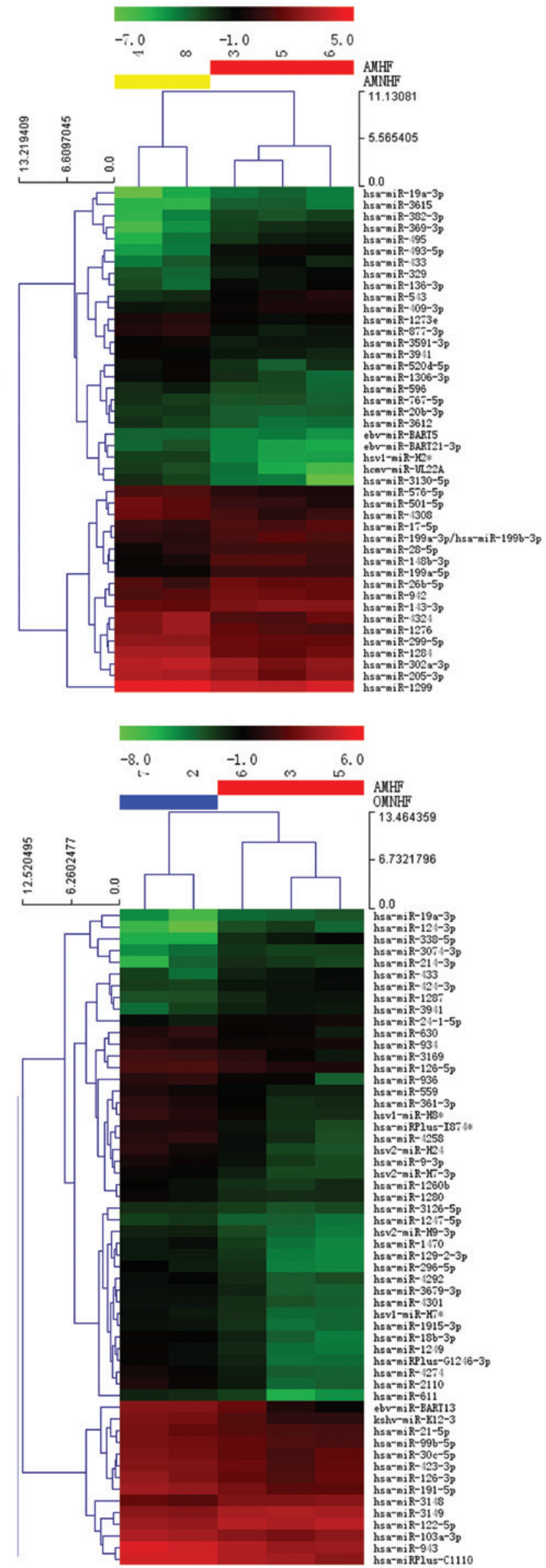
Specifically, it was found that, in the AMHF group, the expression of 17 miRNAs was upregulated and the expression of 21 miRNAs was downregulated by $>1.5$-fold as compared with that in the AMNHF group. Compared with the expression in the $\mathrm{N}$ group, the expression of miRNAs in the AMNHF group was upregulated in 38 and downregulated in 48 cases by $>1.5$-fold. Compared with miRNAs in the OMHF group, 13 miRNAs were upregulated and 43 were downregulated by $>1.5$-fold in the AMHF group.

miRNAs are small, endogenous RNAs that have important roles in living organisms and target mRNAs for degradation or translational repression (6). Dysregulation and tissue-specific patterns of intracellular miRNA expression have been reported for various diseases, in particular for several types of cancer (7). In addition, miRNAs appear to circulate in the blood in a relatively stable form (8), suggesting that miRNAs may have biological functions outside the cell; thus, they can potentially serve as diagnostic or prognostic biomarkers for different types of cancer, as well as therapeutic targets. With regard to cardiovascular diseases, however, the potential of miRNAs as diagnostic markers has only been proposed in the past few years (1), and few prognostic features or therapeutic potentials of circulating miRNAs have been reported. Ai et al (9) found that the miRNA-1 level was significantly higher in plasma from patients with AMI compared with that in patients with no AMI, and the level dropped to normal on discharge following the administration of medication. Increased circulation of miRNA-1 was not associated with age, gender, blood pressure, diabetes mellitus or the established biomarkers for AMI. Wang et al (4) reported that elevated cardiac-specific miR-208a in plasma may be a novel biomarker for the early detection of myocardial injury in humans. miR-208a remained undetectable in patients with no AMI, but was easily detected in $91 \%$ of patients with AMI and in all of the patients with AMI within $4 \mathrm{~h}$ after the onset of the symptoms. Receiver operating characteristic curve analysis of the four miRNAs investigated revealed that miR-208a had the highest sensitivity and specificity for diagnosing AMI. Tijsen et al (10) reported miR423-5p to be a novel circulating biomarker for heart failure. Matsumoto et al (11) found that the serum levels of miR-155 and miR-380 were approximately four- and three-fold higher, respectively, in patients who died within one year subsequent to discharge. Accordingly, a subset of circulating miRNAs may be a predictor of cardiac mortality in patients post-AMI.

In the present study, it was found that, compared with expression in the AMNHF group, the expression of hsa-miR-493-5p, hsa-miR-369-3p, hsa-miR-495, hsa-miR-3615 and hsa-miR-433 was upregulated, whereas that of hsa-miR-877-3p, hsa-miR-1306-3p, hsv1-miR-H2, hsa-miR-3130-5p and hcmv-miR-UL22A was downregulated in the AMHF group; these miRNAs may be novel diagnostic or prognostic biomarkers for heart failure following MI, although they have not been reported previously and the underlying mechanism has yet to be elucidated. The upregulated miRNAs, including hsa-miR-59, hsa-miR-657, hsa-miR-1306-3p, hsv1-miR-H2 and hsa-miR-3130-5p, as well as downregulated miRNAs, including hsa-miR-409-5p,
hsa-miR-24-2-5p, hsa-miR-335-5p, hsa-miR-19a-3p and hsa-miR-130a-3p, may be novel diagnostic or prognostic biomarkers for AMI. Compared with expression in the OMHF group, the expression of hsa-miR-338-5p, hsa-miR-124-3p, hsa-miR-214-3p, hsa-miR-433 and hsa-miR-19a-3p was upregulated, whereas that of hsv2-miR-H24, hsa-miR-1249, hsa-miR-4258, hsa-miR-1470 and hsa-miR-2110 was downregulated in the AMHF group. Significant differences were found in the miRNA expression profiles between patients with different stages of heart failure following MI and the normal control group. Thus, these specific miRNAs may be novel circulating markers for MI and heart failure.

Several limitations of the present study should be mentioned. Firstly, this was an initial screening analysis using a small sample of patients with MI. Secondly, the regulation mechanism and targets of the selected miRNAs were not elucidated. Due to these limitations, further studies with larger sample sizes are warranted to confirm the results of the present study.

\section{Acknowledgements}

The present study was supported by Guangdong Provincial Nature Sciences and Technology Fund (no. S2012010008207) and Foshan Municipal Medical Science and Technology Key Project (no. 201108059).

\section{References}

1. D'Alessandra Y, Devanna P, Limana F, et al: Circulating microRNAs are new and sensitive biomarkers of myocardial infarction. Eur Heart J 31: 2765-2773, 2010.

2. Stoner L, Lucero AA, Palmer BR, Jones LM, Young JM and Faulker J: Inflammatory biomarkers for predicting cardiovascular disease. Clin Biochem 46: 1353-1371, 2013.

3. van Holten TC, Waanders LF, de Groot PG, Vissers J, Hoefer IE, Pasterkamp G, Prins MW and Roest M: Circulating biomarkers for predicting cardiovascular disease risk; a systematic review and comprehensive overview of meta-analyses. PLoS One 22: e62080, 2013.

4. Wang GK, Zhu JQ, Zhang JT, et al: Circulating microRNA: a novel potential biomarker for early diagnosis of acute myocardial infarction in humans. Eur Heart J 31: 659-666, 2010.

5. Kuwabara Y, Ono K, Horie T, et al: Increased microRNA-1 and microRNA-133a levels in serum of patients with cardiovascular disease indicate myocardial damage. Circ Cardiovasc Genet 4: 446-454, 2011.

6. Bartel DP: MicroRNAs: genomics, biogenesis, mechanism, and function. Cell 116: 281-297, 2004.

7. Ueda T, Volinia S, Okumura H, et al: Relation between microRNA expression and progression and prognosis of gastric cancer: a microRNA expression analysis. Lancet Oncol 11: $136-146,2010$.

8. Mitchell PS, Parkin RK, Kroh EM, et al: Circulating microRNAs as stable blood-based markers for cancer detection, Proc Natl Acad Sci USA 105: 10513-10518, 2008.

9. Ai J, Zhang R, Li Y, et al: Circulating microRNA-1 as a potential novel biomarker for acute myocardial infarction. Biochem Biophys Res Commun 391: 73-77, 2010.

10. Tijsen AJ, Creemers EE, Moerland PD, et al: MiR423-5p as a circulating biomarker for heart failure. Circ Res 106: 1035-1039, 2010.

11. Matsumoto S, Sakata Y, Nakatani D, et al: A subset of circulating microRNAs are predictive for cardiac death after discharge for acute myocardial infarction. Biochem Biophys Res Commun 427: 280-284, 2012. 